\title{
Effects of Custom-Made Rigid Foot Orthosis on Pes Planus in Children Over 6 Years Old
}

\author{
Soo-Kyung Bok, MD, Bong-Ok Kim, MD, Jun-Ho Lim, MD, So-Young Ahn, MD
}

Department of Rehabilitation Medicine, Chungnam National University Hospital, Daejeon, Korea

Objective To identify the effects of a custom-made rigid foot orthosis (RFO) in children over six years old with pes planus.

Methods The medical records of 39 children (mean age, $10.3 \pm 4.09$ years) diagnosed with pes planus, fitted with RFOs, and had who more than two consecutive radiological studies were reviewed. The resting calcaneal stance position (RCSP), anteroposterior talocalcaneal angle (APTCA), lateral talocalcaneal angle (LTTCA), the lateral talometatarsal angle (LTTMA), and calcaneal pitch (CP) of both feet were measured to evaluate foot alignment. After diagnosis, children were fitted with a pair of RFOs and recommended to walk with heel strike and reciprocal arm swing to normalize the gait pattern. A follow-up clinical evaluation with radiological measurements was performed after 12-18 months and after 24 months of RFO application. Post-hoc analysis was used to test for significant differences between the radiological indicators and RCSP.

Results With RFOs, all radiological indicators changed in the corrective direction except LTTCA. RCSP and $\mathrm{CP}$ in the third measurement showed significant improvement in comparison with the second and baseline measurements. Additionally, APTCA and LTTMA revealed improvements at the third measurement versus the baseline measurements.

Conclusion This study revealed that radiological indicators improved significantly after 24 months of RFO application. A prospective long-term controlled study with radiographical evaluation is necessary to confirm the therapeutic effects of RFOs and to determine the optimal duration of wear in children with pes planus.

Keywords Foot orthoses, Flatfoot

Received September 23, 2013; Accepted February 17, 2014 Corresponding author: So-Young Ahn

Department of Rehabilitation Medicine, Chungnam National University Hospital, 282 Munhwa-ro, Jung-gu, Daejeon 301-721, Korea Tel: +82-42-338-2460, Fax: +82-42-338-2461, E-mail: asyoung@cnuh. co.kr

(c) This is an open-access article distributed under the terms of the Creative Commons Attribution Non-Commercial License (http://creativecommons. org/licenses/by-nc/3.0) which permits unrestricted noncommercial use, distribution, and reproduction in any medium, provided the original work is properly cited.

Copyright $\odot 2014$ by Korean Academy of Rehabilitation Medicine

\section{INTRODUCTION}

Infants are born with pes planus, otherwise known as flat feet. The medial longitudinal arch of the foot forms naturally during the first decade of life as part of normal development. Volpon [1] reported that most infants rapidly develop a normal plantar arch between 2 and 6 years of age and complete progression occurs by the age of 6 years. Thus, in preschool children, aged 3-6 years, flexible flat foot is observed in $44 \%$ and this decreases to $24 \%$ in children aged over 6 years [2]. However, a small proportion of flat foot cases do not correct with growth 
because of structural abnormalities. This occurs with a prevalence of less than $1 \%$.

There are two types of flat foot: rigid and flexible. Flexible flat foot implies the loss of the longitudinal arch in closed kinetic chain (i.e., weight-bearing) conditions, whereas a rigid flat foot implies the loss of arch height in both open and closed kinetic chain conditions. Abnormal pronation in the subtalar joint can unsettle the midtarsal joint. Additionally, hypermobility, due to abnormal pronation, can lead to complications, such as foot pain, bunion formation, hallux limitus, and hallux rigidus [3]. Otman et al. [4] suggested that flat foot required much more energy expenditure because of the greater muscular effort and instability in this foot condition.

Management of the condition can vary from conservative management to surgical approaches. The latter are rarely used and generally only after failure of conservative management. Conservative (non-surgical) interventions have been reported in the literature and include professional advice, foot orthoses (shoe inserts), stretching exercises, appropriate footwear selection and modifications, activity modifications, manipulation, serial casting, appropriate weight reduction, and anti-inflammatory medications. Surgical interventions include arthroereisis implants, soft tissue medial column reconstruction, reconstruction involving osteotomies, and medial column arthrodesis [5].

Traditionally, children with flat feet have been treated with arch supports or corrective shoes to improve the arch, but recent studies have questioned the effectiveness of these treatments, because development of flat foot is a normal process during early childhood and usually resolves spontaneously without treatment.

Jay et al. [3] reported that there was significant improvement in the resting calcaneal stance position (RCSP) in children aged 20 months to 14 years with flexible flat foot who were provided with a custom-made foot orthosis. In another study on flat foot children, aged 7-11 years, Whitford and Esterman [6] found no evidence to justify the use of orthotic inserts in the management of flexible excess foot pronation in children. However, the previous research used different methods and showed different results according to age or pes planus severity. Moreover, there have been few long-term ( $>24$ months) follow-up studies to date.

Thus, our intent was to conduct a long-term study in individuals with homogenous flat foot after normal development. The aim of this study was to evaluate the effects of a rigid foot orthosis (RFO) on the basis of Blake inverted technique on flexible pes planus in children aged over 6 years through a 24-month follow-up period.

\section{MATERIALS AND METHODS}

In total, 39 children (average age, 10.3 years) diagnosed with flexible flat foot were enrolled. Exclusion criteria included fixed-foot deformity, a previous surgical history, congenital or developmental foot disease, and neuromuscular diseases, such as cerebral palsy. They were evaluated by more than three consecutive radiological studies and by RCSP measurements. Radiological parameters used to evaluate the alignment of both feet included the anteroposterior talocalcaneal angle (APTCA), lateral talocalcaneal angle (LTTCA), lateral talometatarsal angle (LTTMA), and calcaneal pitch (CP). Two rehabilitation medicine physicians recorded the measurements.

RCSP was determined in the following manner. First, subjects lay prone on a bed parallel to the floor. Regardless of the calcaneal fat pad, the upper, middle, and lower bisection points of calcaneus were marked and three points were connected to create a centerline. Then, the subjects stood in a relaxed bipedal stance with their feet apart as wide as an adult's fist. We measured the angle between the centerline of the calcaneus and the vertical line to the ground.

An anteroposterior, lateral weight-bearing radiograph was then obtained for each foot. On the anteroposterior radiograph, one indicator was measured to describe the hind foot alignment. On the lateral radiograph, three indicators were measured to demonstrate the medial longitudinal arch.

Flat foot was defined when either of the feet had $>4^{\circ}$ valgus of RCSP angle and one abnormal radiological finding greater than $30^{\circ}$ in APTCA, $45^{\circ}$ in LTTCA, $4^{\circ}$ in LTTMA, or less than $20^{\circ}$ of CP. The APTCA is the acute angle between the dichotomous line of the calcaneus and talus with the long axis on an anteroposterior radiograph. The LTTCA is the acute angle between the dichotomous line of the calcaneus and talus with the long axis on a lateral radiograph. The LTTMA is the acute angle between the longitudinal axis of the first metatarsus and talus on a lateral radiograph. The $\mathrm{CP}$ is the acute angle between 

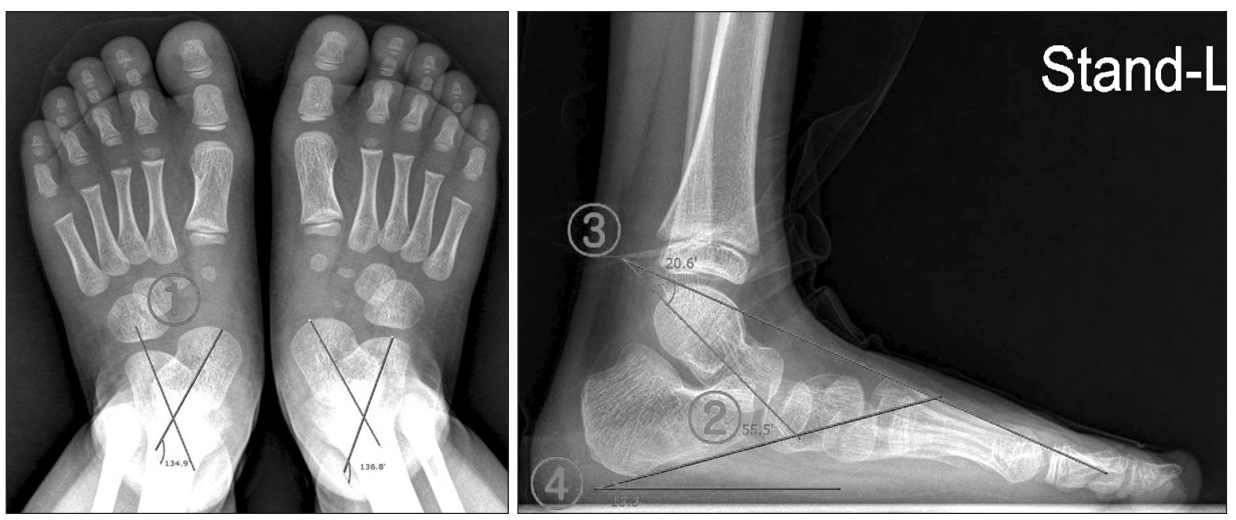

Fig. 1. Anterioposterior view and lateral view of both feet. (1), Anteroposterior talocalcaneal angle; (2), lateral talocalcaneal angle; (3), lateral talometatarsal angle; (4), calcaneal pitch.

the inferior border of the calcaneus and the baseline on a lateral radiograph. The baseline was defined as the connection of the closest point of the first metatarsus and calcaneus on the floor (Fig. 1).

A cast was produced for each subject, from which a pair of RFOs was manufactured (Biomechanics, Goyang, Korea) on the basis of the inverted technique. The orthosis inverts the rear foot and pronates the fore foot through the subtalar joint and longitudinal axis of the midtarsal joint. A $5^{\circ}$ inversion can correct $1^{\circ}$ RCSP in the pronated position. RFOs were produced from a positive cast of the feet, which captured the foot position with the subtalar joint in a neutral position. Each subject was recommended to wear the orthoses for more than 8 hours per day, and to walk with heel strike at initial contact and reciprocal arm swing to normalize the gait pattern. The examination was performed three times: on the first visit, at 12-18 months after application of RFO, and at 24 months after RFOs were provided.

\section{Statistical analysis}

Repeated measures ANOVA was used to compare the follow-up and baseline values. Statistical significance was set at $\mathrm{p}<0.05$. Pearson correlation coefficient was used to assess the correlation between each radiological indicator and RCSP. All analyses were performed using the SPSS ver. 19.0 (IBM, Armonk, NY, USA).

\section{RESULT}

In total, 39 children (18 boys, 21 girls) were included in the study. Their mean age was 10.3 years (range, 6-14 years). At the second measurement, the CP and RCSP values improved significantly in the patients using custommade RFOs. Subsequently, at the third measurement,
APTCA and LTTMA showed improvement; however, there was no significant improvement in the LTTCA value (Fig. 2).

\section{Resting calcaneal stance position}

The baseline, second, and third measurements of RCSP were $-8.0 \pm 5.1,-2.6 \pm 3.2$, and $-1.9 \pm 2.8$, respectively. Comparing the second measurement with the baseline showed a statistically significant improvement. The third measurement showed a statistically significant improvement compared with the second measurement and the baseline value (Table 1 ).

\section{Radiological measurements}

\section{Anteroposterior talocalcaneal angle}

The baseline, second, and third measurements of APT-

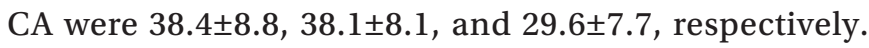
Comparison of the second measurement with the baseline value revealed no statistically significant improvement. The third measurement improved significantly compared with the second measurement and the baseline value.

\section{Lateral talocalcaneal angle}

The baseline, second, and third measurements of LTTCA were $47.3 \pm 6.3,49.8 \pm 7.6$, and $47.3 \pm 5.3$, respectively. There was no statistically significant improvement between the baseline measurement and second measurement. Additionally, comparison of the second and third measurements revealed no statistically significant improvement.

\section{Lateral talometatarsal angle}

The baseline, second, and third measurements of LTTMA were $17.7 \pm 10.0,18.2 \pm 10.4$, and $10.3 \pm 6.4$, respectively. 
(A)

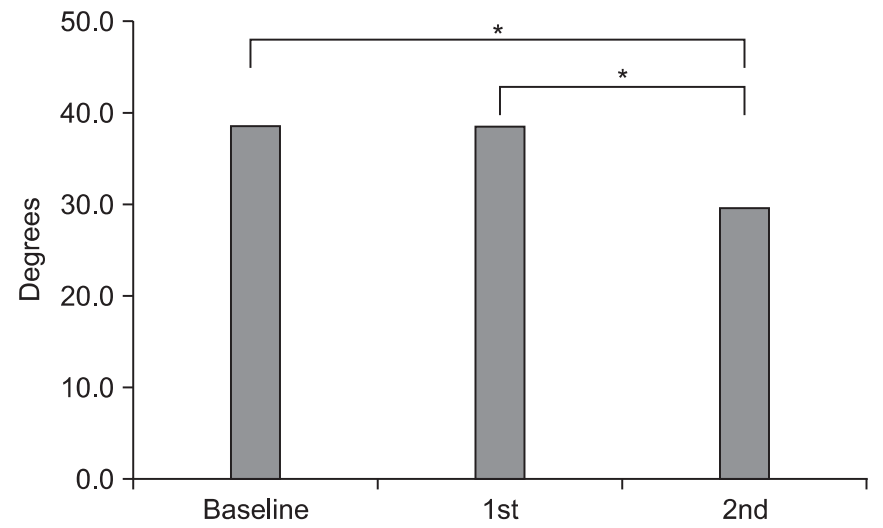

(C)

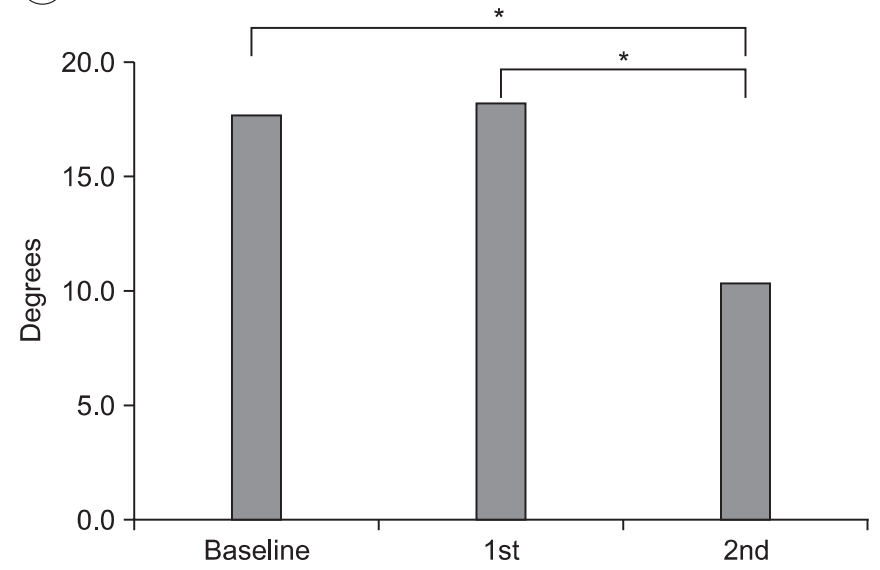

(E)

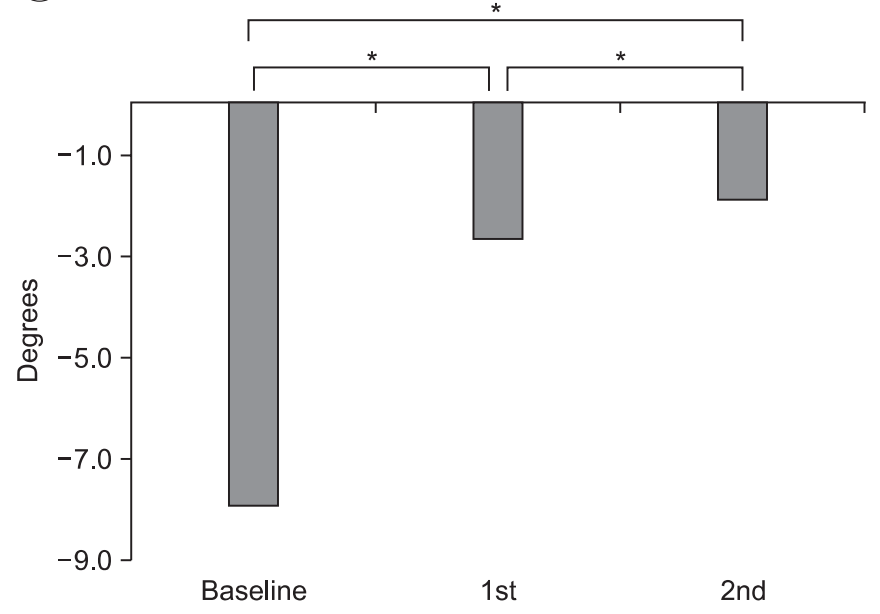

Comparison of values of the second measurement with the baseline values revealed no statistically significant improvement. However, the third measurement showed a statistically significant improvement compared with the second measurement and the baseline value.
(B)

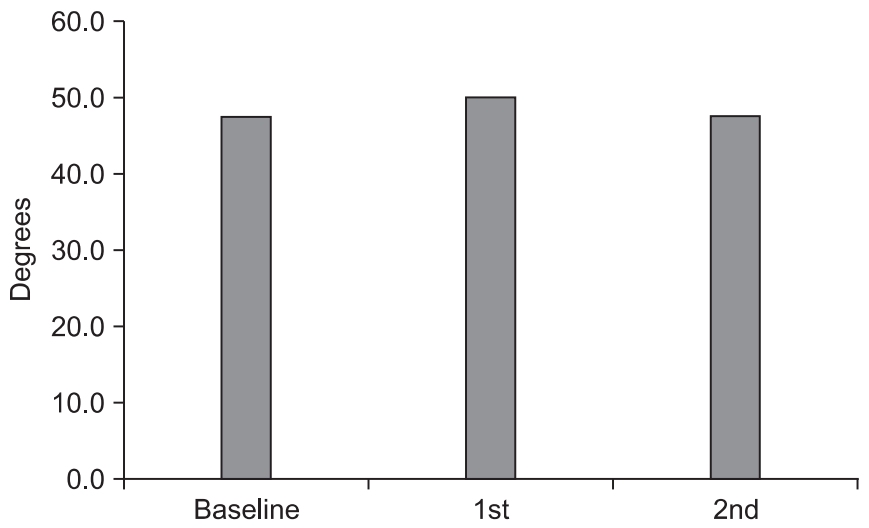

(D)

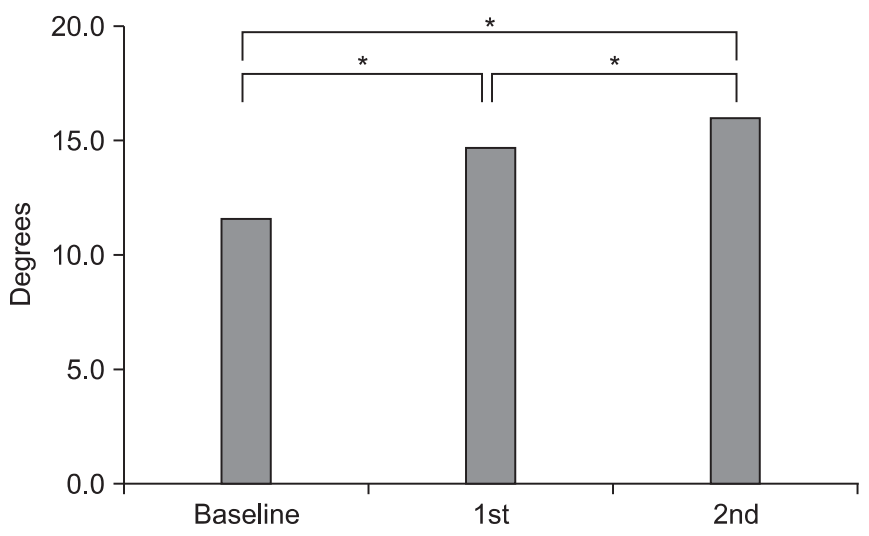

Fig. 2. Serial change in values of anteroposterior talocalcaneal angle (APTCA, A), lateral talocalcaneal angle (LTTCA, B), lateral talometatarsal angle (LTTMA, C), calcaneal pitch (CP, D), and resting calcaneal stance position (RCSP, E). ${ }^{*} \mathrm{p}<0.05$.

\section{Calcaneal pitch}

The baseline, second, and third measurements of $\mathrm{CP}$ were $11.6 \pm 4.7,14.7 \pm 4.6$, and $16.0 \pm 4.4$, respectively. Comparison of the second measurement with the baseline values revealed a statistically significant improvement. 
Table 1. Changes in radiological indicators and RCSP (unit, ${ }^{\circ}$ )

\begin{tabular}{llll}
\hline & Baseline & Second & Third \\
\hline APTCA & $38.4 \pm 8.8$ & $38.1 \pm 8.1$ & $29.6 \pm 7.7^{\mathrm{a}, \mathrm{b})}$ \\
\hline LTTCA & $47.3 \pm 6.3$ & $49.8 \pm 7.6$ & $47.6 \pm 5.3$ \\
LTTMA & $17.7 \pm 10.0$ & $18.2 \pm 10.4$ & $10.3 \pm 6.4^{\mathrm{a}, \mathrm{b})}$ \\
CP & $11.6 \pm 4.7$ & $14.7 \pm 4.6^{\mathrm{c})}$ & $16.0 \pm 4.4^{\mathrm{a}, \mathrm{b})}$ \\
RCSP & $-8.0 \pm 5.1$ & $-2.6 \pm 3.2^{\mathrm{c})}$ & $-1.9 \pm 2.8^{\mathrm{a}, \mathrm{b})}$ \\
\hline
\end{tabular}

Values are presented as mean \pm standard deviations.

RCSP, resting calcaneal stance position; APTCA, anteroposterior talocalcaneal angle; LTTCA, lateral talocalcaneal angle; LTTMA, lateral talometatarsal angle; CP, calcaneal pitch.

a) $<<0.05$ for comparison of the third measurement with the second measurement by post-hoc conferring F-test.

${ }^{b)} p<0.05$ for comparison of the third measurement with the baseline measurement by post-hoc conferring F-test.

${ }^{c)} \mathrm{p}<0.05$ for comparison of the second measurement with the baseline measurement by post-hoc conferring F-test.

Table 2. Correlation coefficient between variables

\begin{tabular}{llllcc}
\hline & APTCA & LTTCA & LTTMA & CP & RCSP \\
\hline APTCA & 1 & 0.377 & 0.998 & -0.735 & -0.594 \\
LTTCA & 0.377 & 1 & 0.433 & 0.351 & 0.521 \\
LTTMA & 0.998 & 0.433 & 1 & -0.692 & -0.544 \\
CP & -0.735 & 0.351 & -0.692 & 1 & 0.982 \\
RCSP & -0.594 & 0.521 & -0.544 & 0.982 & 1 \\
\hline
\end{tabular}

APTCA, anteroposterior talocalcaneal angle; LTTCA, lateral talocalcaneal angle; LTTMA, lateral talometatarsal angle; $\mathrm{CP}$, calcaneal pitch; RCSP, resting calcaneal stance position.

Moreover, the third measurement revealed significant improvements compared with the second measurement and the baseline value.

\section{Correlation}

RCSP showed a strong correlation with CP among the radiological indicators (-0.982) and a moderate correlation with the other indicators. Also, among the radiological indicators, APTCA correlated well with LTTMA (0.998) (Table 2).

\section{DISCUSSION}

Flat foot (pes planus) is a common foot disorder, characterized by flatness of the soles of the feet due to loss of the medial longitudinal arch. This results in overpronation of the subtalar joint. During weight-bearing, pronation is produced by eversion of the calcaneus with simultaneous plantarflexion and adduction of the talus on the calcaneus. Pronation is considered abnormal when it occurs in positions when the foot should be supinating, including during midstance or propulsion.
Methods for correcting flat foot have long been debated, particularly regarding non-surgical interventions. Historically, there have been many studies that have used varying methods of diagnosing the disorder and including the use of various kinds of foot orthoses and footwear. Jay et al. [3] studied the effects of the dynamic stabilizing innersole system in patients with flexible flat foot. Their study was conducted in children aged 20 months to 14 years. After 12 months of using the orthoses, there was significant improvement in RCSP. However, the ages of the subjects covered a broad range, the subjects were not homogeneous, and the follow-up duration was shorter than in our study. Whitford and Esterman [6] evaluated the effects of custom-made and ready-made orthoses in children with flexible excess pronation between the ages of 7 and 11 years. The outcome measures included gross motor proficiency, self-perception, exercise efficiency, and pain after 12 months. This study reported no evidence to justify the use of in-shoe orthoses in the management of flexible foot pronation in children. Although, they limited the subjects to over 6 years, the follow-up duration was relatively short. In our study, with RFOs 
made on the basis of an inverted technique, radiological parameters were improved after 24 months of using the orthoses; they were effective in the development of the medial longitudinal arch in flat foot children over 6 years old. Long-term follow-up may still be needed. Additionally, Wenger et al. [7] performed a study to determine the influence of corrective shoes and inserts for the management of flexible flat foot in infants. Subjects were aged 1-6 years and they underwent treatment for a minimum of 3 years; in this study, wearing corrective shoes or inserts for 3 years did not influence the treatment course of children with flat foot. They said that in children under 6 years old, the medial longitudinal arch may develop spontaneously. Because we agree with the report of Wenger et al. [7] with regard to children younger than 6 years, we studied subjects over 6 years. In this study, RFOs, made on the basis of the inverted technique, were effective in the development of medial arch formation.

The subtalar joint has been described as a triplanar joint. It involves motion in the sagittal, transverse, and frontal planes. Two-thirds of this total motion is in the direction of supination, whereas one-third is in the direction of pronation. The normal subtalar neutral position is $0^{\circ}$. In this study, we used orthoses according to Blake inverted orthotic technique. Blake and Ferguson [8] and Blake [9] applied inverted functional orthoses for the management of severe flat foot and sports-related complications, such as medial knee pain. The control area of the inverted orthotic device is the medial calcaneal area. The orthosis can supinate the rear foot, by inverting the calcaneus, resulting in dorsiflexion and abduction of the talus. Because it does not rely on inversion of the fore foot on the rear foot, it is possible to produce a greater supinatory force on the rear foot. Additionally, the medial arch filling maintains the relationship of the fore foot and rear foot, parallel to the supporting surface.

The RCSP represents the frontal plane position of the calcaneus. The radiological measures of medial longitudinal arch structures were defined as the gold standards by Saltzman et al. [10]. These radiological measures are the LTTCA, the lateral talo-first metatarsal angle, the talo-horizontal angle, and the CP angle. The LTTCA and $\mathrm{CP}$ angles represent the alignment of the hind foot in the sagittal plane position. Additionally, the talar inclination can be measured by determining the talo-first metatarsal angle. Increased talar inclination causes the subtalar joint to pronate; thus, a larger area of the midfoot comes in contact with the ground. Unlike the lateral view, in the anteroposterior view, the talocalcaneal and talometatarsal angles represent alignment of the hind foot and midfoot in each of the transverse plane positions

Our study demonstrated the effects of the custom-made RFOs on the position change of the talus and calcaneus, allowing the development of the medial arch in flexible flat foot. At the second measurement, CP and RCSP improved significantly compared with baseline. At the third measurement, 24 months after RFO application, APTCA, LTTMA, CP, and RCSP had improved significantly compared with the second measurement and with baseline values. With regard to APTCA and LTTMA, at the third measurement, significant improvement was observed compared with the baseline and second measurement values. However, LTTCA revealed no significant improvement in the follow-up measurements.

Because the control target of the inverted technique is the medial side of the calcaneus, there initially seems to be a change in the hind foot, which is the position of the sagittal and frontal planes of the calcaneus. However, if the foot orthoses are used for more than 24 months, there could be a change in the position of the transverse plane (APTCA) and the sagittal plane of the talus. The more talar inclination is increased, the more there seems to be development of the medial longitudinal arch because of midfoot molding.

LTTCA revealed no significant improvement at the second or third measurements compared with the baseline values. The resulting values of LTTCA were unexpected and do not support our initial hypothesis.

There was a major limitation in the current study. Specifically, there was no control group. Because changes in radiological parameters occur normally in childhood, a control group is required. We considered this, but ethical problems occurred in enrolling subjects into the control group. Because this study was conducted in the clinical setting, not in a laboratory, patients' parents did not agree that their children would be enrolled to the control group. Also, from 10 years of practice experience at our clinic, we are convinced of the beneficial effects of foot orthoses. In reviewing articles regarding flat foot from 1980 to 2012, only three studies had control groups: Whitford and Esterman [6], Gould [11], and Wenger et al. [7]. Moreover, Whitford and Esterman [6] was the only 
age-controlled study (subjects $>6$ years old). Indeed, recruitment of a control group is a complex problem. To minimize the effects of spontaneous improvement in foot arch, we only enrolled children over 6 years old.

This study suggests that radiological indicators improved significantly after 24 months of RFO use. At the second measurement, CP and RCSP had improved significantly compared with those at baseline. At the third measurement, after 24 months of RFO application, APTCA, LTTMA, CP, and RCSP improved significantly compared with the second measurements and the baseline values. However, LTTMA showed no significant improvement during follow-up.

We conclude that RFOs fabricated with the inverted technique can be effective devices for treating flexible flat foot in children over 6 years old. A prospective long-term study with radiographical measurements is necessary to confirm the therapeutic effects of RFOs and to determine the optimal duration of wear in children with pes planus.

\section{CONFLICT OF INTEREST}

No potential conflict of interest relevant to this article was reported.

\section{REFERENCES}

1. Volpon JB. Footprint analysis during the growth period. J Pediatr Orthop 1994;14:83-5.

2. Pfeiffer M, Kotz R, Ledl T, Hauser G, Sluga M. Preva- lence of flat foot in preschool-aged children. Pediatrics 2006;118:634-9.

3. Jay RM, Schoenhaus HD, Seymour C, Gamble S. The Dynamic Stabilizing Innersole System (DSIS): the management of hyperpronation in children. J Foot Ankle Surg 1995;34:124-31.

4. Otman S, Basgoze O, Gokce-Kutsal Y. Energy cost of walking with flat feet. Prosthet Orthot Int 1988;12:736.

5. Evans AM, Rome K. A Cochrane review of the evidence for non-surgical interventions for flexible pediatric flat feet. Eur J Phys Rehabil Med 2011;47:69-89.

6. Whitford D, Esterman A. A randomized controlled trial of two types of in-shoe orthoses in children with flexible excess pronation of the feet. Foot Ankle Int 2007;28:715-23.

7. Wenger DR, Mauldin D, Speck G, Morgan D, Lieber RL. Corrective shoes and inserts as treatment for flexible flatfoot in infants and children. J Bone Joint Surg Am 1989;71:800-10.

8. Blake RL, Ferguson H. Foot orthosis for the severe flatfoot in sports. J Am Podiatr Med Assoc 1991;81:549-55.

9. Blake RL. Inverted functional orthosis. J Am Podiatr Med Assoc 1986;76:275-6.

10. Saltzman CL, Nawoczenski DA, Talbot KD. Measurement of the medial longitudinal arch. Arch Phys Med Rehabil 1995;76:45-9.

11. Gould N, Moreland M, Alvarez R, Trevino S, Fenwick J. Development of the child's arch. Foot Ankle 1989;9:241-5. 